\title{
Raising standards in microbiome research
}

\author{
If the vast potential for microbiome research is to be translated into scientific advances and real world \\ applications, the development of standard operating procedures will be necessary to ensure reproducibility \\ and gain regulatory approval. However, standards should not come at the expense of innovation.
}

With the Human Microbiome Project and MetaHIT both conceived in 2007, it can reasonably be claimed that the modern era of the human microbiome field will soon complete its first decade. And what a decade it has been. Interest is booming in all types of microbiome research, whether host-associated or environmental, as researchers from across the life and earth sciences become increasingly aware of the fundamental role that microorganisms have in organismal health and ecosystem function. As demonstrated by the recent launch of the US National Microbiome Initiative (NMI), a program aiming to martial federal and private funding for the integrated study of microbiomes across different ecosystems, microbiology is once again occupying the centre stage.

With this new-found attention comes opportunity, not only for improving our understanding of the diversity and function of microorganisms inhabiting different niches, but also to develop new applications to benefit health, agriculture and the environment. In human health, the potential for microbiomebased therapies, diagnostics and dietary modifications are gaining increasing attention, as an ever growing list of diseases and conditions are shown to be impacted by our microbial flora. In agriculture, understanding the role of the soil and plant-associated microbiome in growth and resilience of crops has the potential to improve yields and help to feed an expanding global population. Our growing appreciation of how microbial communities respond to perturbations, such as the release of millions of gallons of crude oil and chemical dispersants, can help to inform future policy in responding to environmental catastrophe. Translating such vast potential into real world applications will not happen overnight, but as new products and tools emerge in the coming decades, they will need suitable regulatory frameworks to make certain that they are effective and safe to use. For example, faecal microbiota transplantation (FMT) has proven to be highly effective in treating life-threatening infection with the enteric bacterial pathogen Clostridium difficile. However, it is less effective in treating other chronic enteric conditions, and the unregulated use of FMT brings with it the potential for transferring potential pathogens.

A necessary step in establishing the requisite regulation through which to bring microbiome products to market, will be the adoption of standard operating procedures (SOPs) for interrogating the microbiome and for assessing the impact of a therapy or tool. Moreover, beyond simply being a necessary part of commercialization, the adoption of SOPs can have an important role in driving reproducibility and enabling comparison between studies. For example, human gut microbiota data can be substantially altered by choices in experimental approach as well as by the analytical pipeline adopted. Furthermore, without rich metadata providing information on antibiotic (or other therapeutic) interventions, dietary intake and a range of other markers of patient health, attempts to draw comparison between datasets generated from different cohorts can also be confounded.

The field has already begun to embrace the need for developing standards at a variety levels. For example, The International Human Microbiome Standards (IHMS) project was established to develop SOPs to optimize data quality and comparability for the metagenomic analysis of human faecal material. The IHMS assessed a suite of sample collection approaches and then used standard samples (stool and bacterial mix) to allow comparison of in-house protocols for DNA extraction and sequencing from laboratories worldwide, revealing key steps in storage and sample processing that altered the data output. It culminated in the publication of 14 SOPs covering all stages of the process, including metadata description, sample collection, DNA extraction, sequencing approach and data analysis. Although these SOPs represent an important step in establishing the necessary international standards, ensuring that such SOPs are adopted widely by academic and industrial researchers will be a challenge. Furthermore with a focus on stool, these SOPs are therefore validated for only a fraction of the types of sample and analysis of interest to the wider microbiome research community. Variation in microbial density and in the physical and chemical makeup of the substrate in diverse sample types will impact upon the suitability of any given methodological approach. Efforts similar to the IHMS will need to be established for a range of sample types including human tissues other than stool, for animal models and for those from marine, terrestrial and built environments. In this regard, the need to develop reference samples, data and methods for microbiome analysis are already on the agenda for discussion by a number of bodies, including the US National Institute of Standards and Technology who will host a workshop dedicated to this issue in the next few weeks.

As important as developing SOPs will be for reproducibility and regulation, there are concerns that trying to enforce the adoption of a limited set of experimental and analytical protocols could stifle innovation in the basic research laboratory. In a field where technology is advancing so rapidly, why should researchers adopt methodology that might not be best optimized to assess their given samples or biological questions? For example, soil physiochemical properties can vary dramatically between environments and a protocol optimized to assess one type may not be most suitable for other samples. Similarly, as new sequencing technologies come online, pre-existing SOPs may rapidly find themselves outdated. Here, benchmarking new approaches against reference samples, datasets and protocols will likely be the answer, but what such reference materials should be remains to be determined.

Balance needs to be struck then, between allowing for technological and scientific innovation and creativity in the basic research laboratory, and adoption of SOPs and benchmarking to provide greater comparability and reproducibility between studies. Importantly, this cannot be addressed post-hoc but instead needs to be considered at the inception of a study programme, suggesting a potential role for those responsible for funding microbiome research in assessing the balance between adoption of internationally agreed SOPs and the specific nature of a given study. Despite the progress to date, the microbiome field remains in the foothills, and reaching what to many people may have seemed like a summit has simply revealed the far higher peaks beyond and the challenges faced in scaling them. 